\title{
Electrically Controlled Waveguide Liquid-Crystal Elements ${ }^{\pi}$
}

\author{
O. S. Kabanova, E. A. Melnikova*, I. I. Olenskaya, and A. L. Tolstik \\ Belarusian State University, Minsk, 220030 Belarus \\ *e-mail:melnikova@bsu.by \\ Received March 5, 2014
}

\begin{abstract}
Liquid-crystal (LC) elements with an electrically controlled spatial topology of director orientation have been developed and manufactured. Waveguide light-beam propagation mode in a spatially structured LC cell has been realized for the first time; the possibility of creating electrically controlled waveguide dividers and adders has been demonstrated experimentally.
\end{abstract}

DOI: $10.1134 / \mathrm{S} 1063785014070207$

An abnormally high optical anisotropy of liquid crystals (LCs) and the possibility to control anisotropy in thin-film LC elements by low voltages (on the order of several volts) offer great prospects in the development of novel methods and devices for the control of light fields on the basis of compact LC elements. In addition to conventional applications (indicators, light modulators, displays), this work presents an analysis of the approaches to the creation of LC devices intended to control the light beams (phase plates, deflectors, diffraction LC elements) [1-8]. In recent works, light-beam propagation in spatially structured nematic LCs has been considered. The possibility of refraction, reflection, and polarization separation of light beams at the interface of two LC domains has been demonstrated [9-11], and the feasibility of lightinduced waveguide propagation of laser radiation and of nematicon formation has been suggested [12-14].

This work is devoted to development of electrically controlled, spatially structured LC elements and to realization of waveguide-propagation and light-beam control regimes based on them.

The structure of an electrically controlled waveguide LC element is shown in Fig. 1a. This LC element includes two glass substrates $l a$ and $1 b$ with surface electrodes. On the first substrate $1 a$, transparent electrode $2 a$ was formed of indium oxide, and spatially structured electrode $2 b$ of chromium on the second substrate $1 b$ was manufactured by the optical lithography method with the use of the specially developed templates. The orienting photopolymeric coating $3 a$ and $3 b$ was applied to the substrates with the electrodes and rubbed to give the director a planar orientation. The thickness of the LC layer in a waveguide element was dictated by the spacers and came to $20 \mu \mathrm{m}$. This work was performed with positive birefringent nematic liquid crystal 4 having refractive index anisotropy 0.18 .

\footnotetext{
TThe article was translated by the authors.
}

The LC cells enabling realization of the waveguidepropagation and light-beam separation modes were manufactured with the electrode structures shown in Figs. 1b ("forks") and 1c ("double forks"). The electrodes were 40 and $20 \mu \mathrm{m}$ thick, respectively; a distance between the electrodes at the input was 270 (Fig. 1b) or $520 \mu \mathrm{m}$ (Fig. 1c). The electrode length to forking was $3500 \mu \mathrm{m}$. After forking, the distance between the electrodes ("fork" width) was $100 \mu \mathrm{m}$.

The formation of the controlled LC waveguides is based on realization of the effect of total internal reflection from two LC regions with different topologies of the director orientations [10]. When no voltage is applied to the LC divider, a liquid crystal is planar oriented over the whole volume of the element. With the voltage applied, due to the Fredericks transition, molecules of LC are oriented with the electric-field lines (homeotropic orientation), the spatial distribution of which within the LC volume is determined by topology of the structured electrode. In the areas of an LC element without the electrode, the director is planar oriented. As a result, the director orientation is spatially modulated within the LC cell volume and followed by modulation of the LC refractive index anisotropy. In the case of linearly polarized light, such a structure may represent a waveguide element. Indeed, when the electric vector of a linearly polarized light wave is coincident with the direction of a homeotropically oriented liquid crystal, the refractive index for such polarization mode is determined by the refractive index of an extraordinary wave $\left(n_{\mathrm{e}}=1.67\right)$. In the areas, where there is no electrode and the LC director retains its initial planar orientation, the refractive index is governed by that of an ordinary wave $\left(n_{\mathrm{o}}=1.49\right)$. In this way, under an electric field, for polarization of the light wave coincident in its direction with the homeotropic orientation of LC and for the propagation angles below $25^{\circ}$, the total internal reflection conditions and waveguide propagation of laser radiation are realized. 
(a)

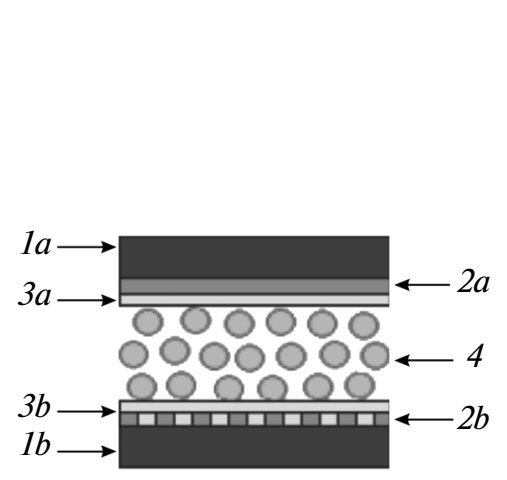

(b)

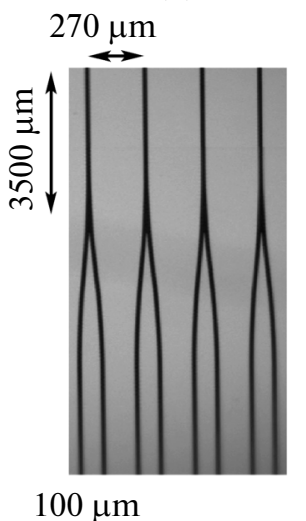

$100 \mu \mathrm{m}$ (c)

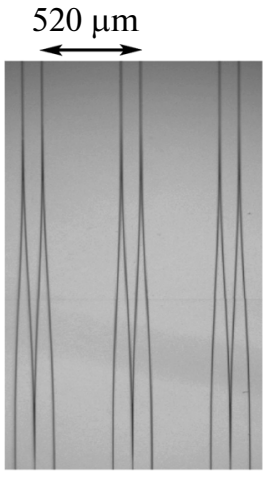

Fig. 1. (a) Schematic diagram of LC cell and (b, c) fork and double-fork electrode configurations, respectively.

(a)

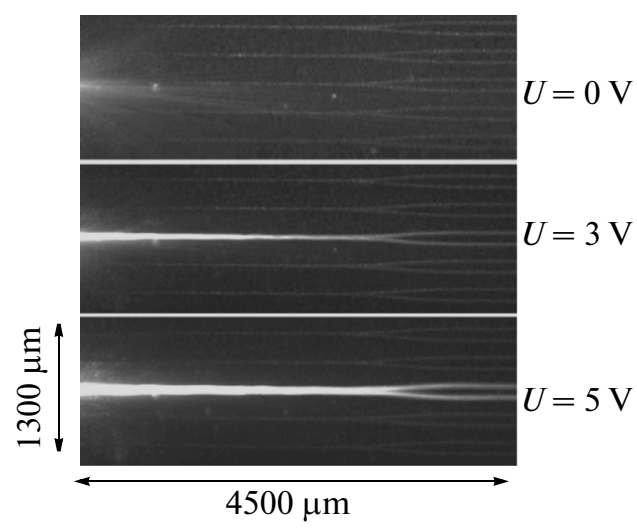

(b)

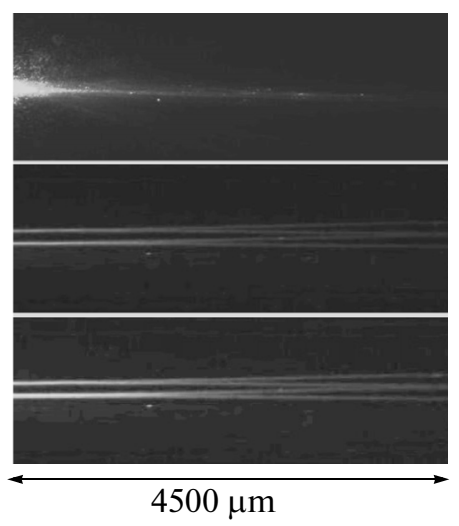

Fig. 2. Propagation of laser radiation in LC cells with (a) fork and (b) double-fork electrode configurations.

The propagation pattern of laser radiation in the manufactured LC elements is shown in Fig. 2. The linearly polarized laser radiation of a $\mathrm{He}-\mathrm{Ne}$ laser was injected into the end of an LC element by means of a micro-objective, with light propagating in the plane of an LC capillary within the limits of one electrode. Without the field applied (Fig. 2, $U=0 \mathrm{~V}$ ), the light injected into an LC cell is scattered by inhomogeneities of the liquid crystal. Application of an electric field results in excitation of the waveguide channels within the medium volume and laser radiation propagates along these channels (Fig. 2, $U=3$ and $5 \mathrm{~V}$ ). As seen in Fig. 2a, the light beam is captured by a single waveguide and then is forked due to the electrode structure. In the case of double forks, radiation is captured by both waveguides and forked too (Fig. 2b). The two beams merge at the junction of two internal electrodes. Two outer beams at the output carry information on the input signals used, the central beam being associated with the total optical signal.

It should be noted that an increase in the voltage amplitude applied to the LC element leads to the increased propagation length of a light beam within the LC cell (Fig. 2). This stems from the fact that the LC director orientation at low voltages occurs only at the central part of an LC layer, far from the substrates. The increased voltage results in growing of the LC layer region from the center to the substrates, where the director reorientation takes place. This means that, with an increase in the applied voltage, the total internal reflection condition and waveguide propagation of laser radiation will be encountered for an evergrowing part of the beam.

In the process of this work, a technology of LC elements with different topological structures has been developed and the feasibility of electrically controlled waveguide structures has been demonstrated. The manufactured spatially structured LC elements exhibiting the functions of waveguide dividers and adders may be used as prototypes of the electrically controlled (active) components for fiber-optic communication lines. 


\section{REFERENCES}

1. T. Mutsui, M. Ozaki, and K. Yoshino, J. Opt. Soc. Am. 21, 1651 (2004).

2. P. Pagliusi and G. Gipparrone, J. Opt. Soc. Am. 21, 996 2004).

3. T.-H. Lin and A. Y.-G. Fuh, Opt. Lett. 30, 1390 (2005).

4. L. Song and W.-K. Lee, Opt. Commun. 259, 293 (2006).

5. O. Trushkevych, N. Collings, W. A. Crossland, and T. D. Wilkinson, Appl. Opt. 45, 8889 (2006).

6. A. A. Kazak, E. A. Melnikova, A. L. Tolstik, V. V. Mahilny, and A. I. Stankevich, Tech. Phys. Lett. 34, 861 (2008).

7. A. A. Kazak, A. L. Tolstik, and E. A. Melnikova, J. Opt. Technol. 77, 461 (2010).
8. A. S. Ostrovsky, C. Rickenstorff-Parrao, and V. Arriz'on, Opt. Lett. 38, 534 (2013).

9. M. Peccianti, A. Dyadyusha, M. Kaczmarek, and G. Assanto, Nature Phys. 2, 737 (2006).

10. A. A. Komar, M. A. Kurochkina, A. A. Melnikova, A. I. Stankevich, and A. L. Tolstik, Tech. Phys. Lett. 37, 704 (2011).

11. A. A. Kazak, A. L. Tolstik, E. A. Melnikova, and A. A. Komar, Int. J. Nonlin. Phenom. Complex Syst. 16, 302 (2013).

12. G. Assanto, A. Fratalocchi, and M. Peccianti, Opt. Express 15, 5248 (2007).

13. A. G. Maksimochkin, S. V. Pasechnik, G. I. Maksimochkin, and V. G. Chigrinov, Opt. Commun. 283, 3136 (2010).

14. A. Piccardi, A. Alberucci, and G. Assanto, Int. J. Mol. Sci. 14, 19932 (2013). 